\section{Combined 23-gauge microincisonal \\ vitrectomy \\ surgery and \\ phacoemulsification \\ with AcrySof toric \\ intraocular lens \\ implantation: a \\ comparative study}

Eye (2011) 25, 1387; doi:10.1038/eye.2011.195

Correction to: Eye (2011) 25, 1327-1332; doi:10.1038/

eye.2011.168; published online 15 July 2011

Since the publication of the above article, the authors have noticed that the diopter (D) range given for spherical IOL correction in the third paragraph of the Patients and methods section was incorrect. The correct details are given below.
DH Park, JP Shin and SY Kim

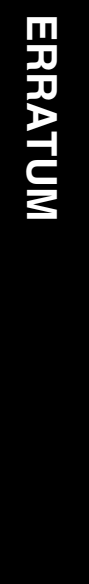

The inclusion criteria were as follows: patients with cataract and vitreoretinal diseases requiring vitrectomy; age between 60 and 85 years; preoperative regular corneal astigmatism >1D; need for spherical IOL correction between 16 and 25 D; and pupil dilation $\geq 6 \mathrm{~mm}$.

The typesetters would like to apologise for this mistake. 